\title{
Los fenómenos de imitación en las viviendas urbanas romanas en el Nordeste de la Península Ibérica
}

\author{
The Roman urban household: imitation phenomena \\ in the Northeast of the Iberian Peninsula
}

\author{
Paula URIBe Agudo*
}

\begin{abstract}
RESUMEN
Presentamos en este trabajo cuatro ejemplos pertenecientes a la edilicia doméstica romana, situados en el cuadrante del nordeste peninsular, que evidencian la temprana imitación por parte de las elites indígenas de los modelos

romanos.
\end{abstract}

\section{PALABRAS CLAVE:}

Edilicia doméstica romana, imitación, Azaila, La Caridad, Caesar Augusta, Bilbilis, balneum, triclinium, patios porticados.

\begin{abstract}
The aim of this paper is to present four examples of Roman households which demonstrate the early adoption of the Roman modus vivendi by the Iberian indigenous population. The examples offered are located at the North-eastern part of the Iberian Peninsula.

KEYWORDS:

Roman household, imitation, Azaila, La Caridad, Caesar Augusta, Bilbilis, balneum, triclinium, porticated courtyard.
\end{abstract}

Abordamos en este trabajo los fenómenos de imitación desde el punto de vista de la edilicia doméstica urbana en época romana. Este tipo de restos arqueológicos constituyen un baremo de excepción para medir el grado de adopción del modo de vida romano adoptado en el cuadrante nordeste de la Península lbérica desde finales del siglo I a.C. Obviamente, en el caso de la edilicia doméstica no se imitan únicamente determinados objetos, prácticas o costumbres. En realidad, al poseer o vivir en una casa 'a la romana' —es decir, con patio central, tablinum, tri-

* Departamento de Ciencias de la Antigüedad, Facultad de Filosofía y Letras, Universidad de Zaragoza (C/ Pedro Cerbuna, 12. 50009 Zaragoza). E-mail: uribe@ unizar.es.

Artículo basado en la comunicación leída el 19 de Mayo de 2008, en la VII edición del Encuentro de Jóvenes Investigadores de Historia Antigua de la Universidad Complutense de Madrid. 
clinum, etc.- se está imitando no sólo un modelo arquitectónico, sino el modelo de vida del colonizador que en definitiva fue el modus vivendi romano.

De esta manera, en primer lugar, planteamos cuatro ejemplos que, según nuestra opinión, mejor ilustran la mimesis o los procesos de imitación, para finalmente extraer una serie de conclusiones relacionadas con el debate final expuesto en la mesa redonda.

\section{AZAILA (CABEZO DE ALCALÁ, TERUEL)}

En este sentido, cabría hacer referencia, en primer lugar, al pequeño oppidum situado en el cerro de Alcalá (Azaila, Teruel) que M. Beltrán (1976) situó cronológicamente entre el VII a.C. y el primer cuarto del I a.C. La antigua ciudad, con unas 20 hectáreas, se constituyó como el claro binomio entre acrópolis y ciudad baja. Esta última, con unos límites poco claros, albergó en su zona de servicios las supuestas termas públicas. Por otro lado, en la acrópolis amurallada se situaron, aparte de diferentes viviendas, un pequeño templo in antis de orden toscano. Por lo tanto, a pesar de esta morfología de características indígenas, contó con equipamientos públicos netamente romanos, y sobre todo tres viviendas que destacan sobre las demás - las viviendas $8 \mathrm{~A} / \mathrm{B}, 5 \mathrm{C}$ y $2 \mathrm{D}$ - por presentar un plan de tipo claramente mediterráneo, caracterizado por la organización de las estancias en torno a un patio central. Además, estas viviendas contaron con otro tipo de infraestructuras, como el hortus y el amplio vestíbulo de la vivienda $5 \mathrm{C}$, que las separan estructuralmente del resto de las viviendas indígenas del oppidum.

Respecto a su decoración, no conocemos ningún tipo de pavimento hallado in situ, únicamente existen noticias sobre algunos fragmentos de opus signinum, conservados en el Museo de Teruel. Las pinturas halladas en la vertiente sur de la acró-

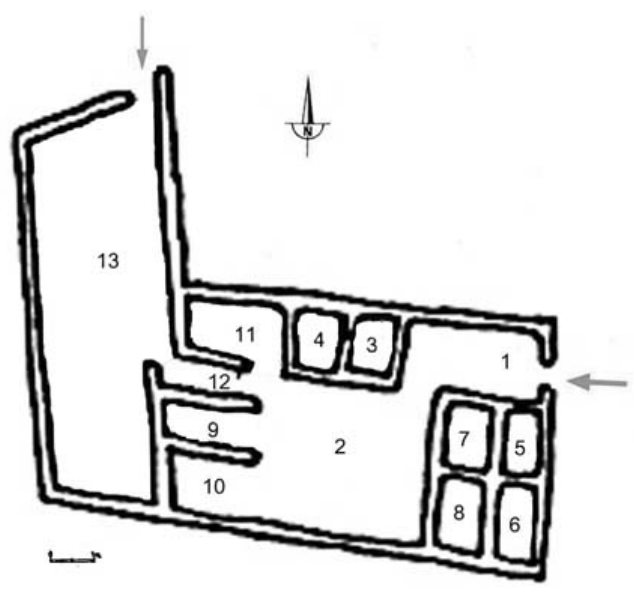

Fig. 1. Plano de la vivienda $5 C$ de Azaila (a partir de M. Beltrán 1991). 


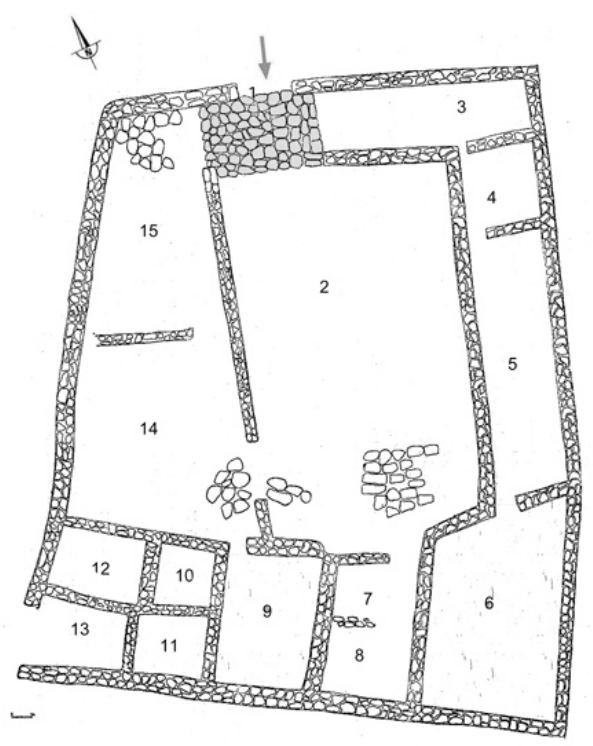

Fig. 2. Plano de la vivienda 2D de Azaila (a partir de M. Beltrán 1991).

polis pudieron pertenecer al I estilo, debido a que en ellas aparecen representadas decoraciones marmóreas con imitación de alabastro, brechas y cubos en perspectiva datadas en el primer cuarto del siglo I a.C. por M. Beltrán (1976: 145).

\section{LA CARIDAD (CAMINREAL, TERUEL)}

Quizás el ejemplo más conocido sea la Casa de Likine del yacimiento de La Caridad de Caminreal, también en Teruel. Se trataría de una ciudad, todavía de nombre desconocido, fundada ex novo y construida siguiendo un plan regular determinado por calles ortogonales. La fiabilidad de su documentación arqueológica y la morfología del edificio representan uno de los ejemplos más claros de la adopción de los modelos de vida romanos por parte de las elites locales.

De este modo, el ejemplo temprano de la 'Casa de Likine' de Caminreal —desde finales del II a.C. hasta primer tercio del I a.C. (Vicente et alii 1989: 30)—, con un espacio central porticado, sin impluvio ni cisternas documentadas y una habitación de aparato en posición central, se convierte en un modelo simplificado de la casa de peristilo desarrollado posteriormente en la Península¹. Dentro de ella se

1 En definitiva, como planteaba Gros, Likine es una síntesis precoz de los elementos de la casa itálica con su axialidad y de la casa helenística con el cuadripórtico, constituyéndose en un importante jalón en la difusión de las grandes domus axiales de peristilo que aparecen en todo el imperio a partir de época flavia. De similares características mencionaríamos la Casa VI Des Antes de Glanum (mediados del I a.C.) 


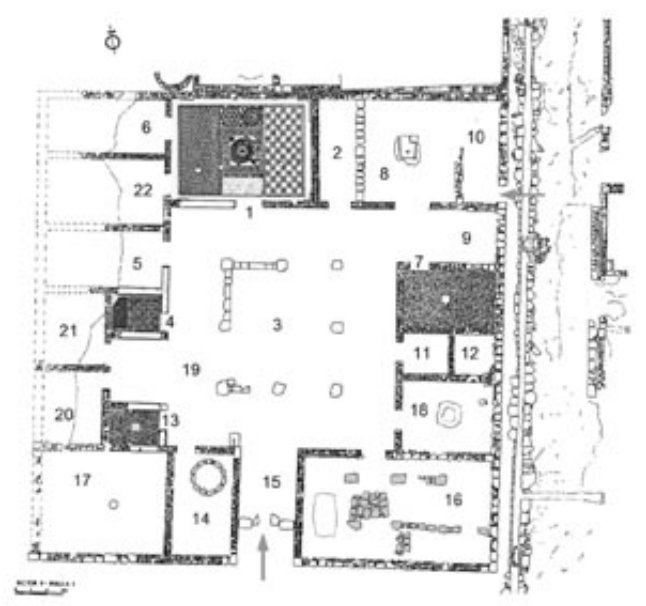

Fig. 3. Plano de la Casa de Likine, Caminreal (a partir de Vicente et alii 1991).

documentan ya repertorios decorativos totalmente itálicos que configuran los cubicula y la estancia central, famosa por su inscripción con signario ibérico². Para Vicente (1993: 755) el sustantivo personal Likine se interpretaría, no como el musivario, sino como el propietario, además de considerarlo un íbero debido a que en el resto de inscripciones aparecidas en la vivienda estuvieron realizadas en ibérico y sólo un sello de un mortero fue realizado en latín. Asimismo, cabría considerar que el modo de vida que se refleja en esta casa no pudo desarrollarse sin la posesión de unos medios económicos notables que, en este caso, pudieron proceder fundamentalmente de la explotación agrícola del territorio.

El análisis detallado del material hallado ${ }^{3}$ en la vivienda de Caminreal y un estudio en profundidad de los espacios, permiten a Beltrán (2003: 31) dividir la vivienda entre habitaciones de representación y espacios productivos -8, 14, 16 y 18-, comparando estos últimos con la parte correspondiente a la pars fructuaria de una villa. En nuestra opinión, no debe sorprender que en las viviendas urbanas, pertenecientes sobre todo a estas fechas, se combinen espacios productivos con habitaciones de representación. Un ejemplo claro que demuestra estos aspectos se hallaría en la vivienda de Moltone de Tolva, en la Lucania interior, que pone en

distribuida en torno a un pórtico en forma de pastas y con una antigua habitación en su eje axial que desempeñaría el papel de tablinum con la inscripción Co. Sulla en su pavimento atribuida a su propietario Cornelio Sulla (Gros 2001: 146).

2 l.i.ki.n.e.te. e.ki.a.r. u. se.ke.r.te.ku. (Vicente et alii 1993: 755).

${ }^{3}$ Se recuperaron una gran cantidad de materiales arqueológicos de los que no existe un reparto selectivo según la función del espacio. La distribución de las herramientas, molinos, cerámicas, armas e instrumentos domésticos muestra una mezcla constantemente repetida de elementos considerados de lujo con herramientas agrarias o artesanales. Parece pues constatarse una modificación de uso respecto a la función teórica de las diferentes estancias. Por otra parte, la acumulación de objetos tan diversos puede resultar excesiva en consonancia con las necesidades de los ocupantes (Vicente et alii 1991). 


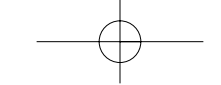

Los fenómenos de imitación en las viviendas urbanas romanas en el Nordeste...

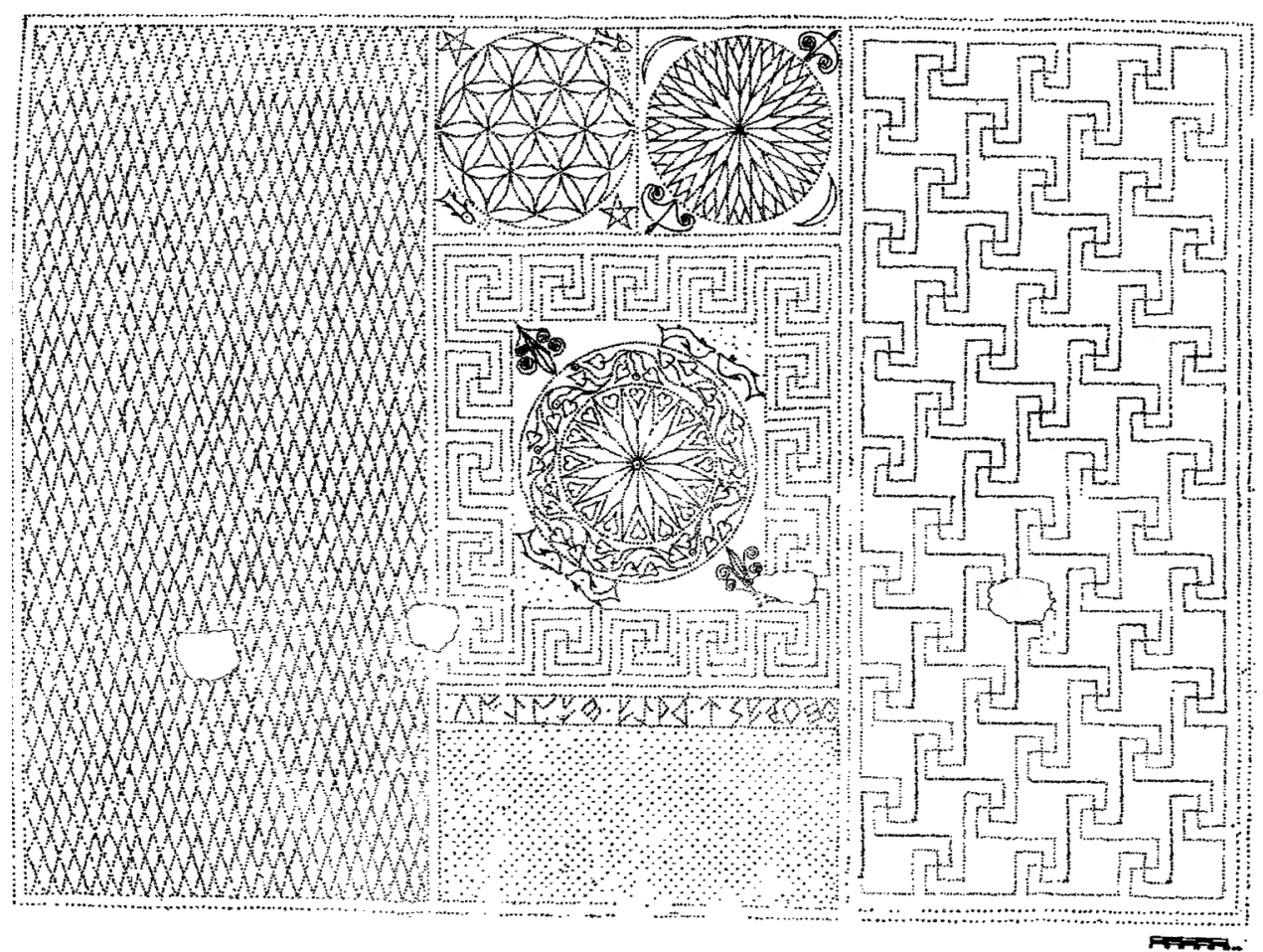

Fig. 4. Opus signinum de la estancia 1 de la Casa de Likine, Caminreal (a partir de Vicente et alii 1991).

evidencia las transformaciones acaecidas en las residencias indígenas con la llegada de los romanos (Russo 1996: 85).

\section{CAESAR AUGUSTA (ZARAGOZA)}

Otro ejemplo, en este caso no una vivienda excavada en su totalidad sino un hallazgo fruto de las intervenciones arqueológicas en la ciudad de Zaragoza, es el opus signinum exhumado en la C/Don Juan de Aragón. Siguiendo los planteamientos de Fatas y Beltrán (1998: 10), la ciudad de Caesar Augusta sería fundada en torno a los años 15-14 a.C. por Augusto para asentar a los veteranos de las legiones IV, VI y X. Situada en un punto estratégico del valle del Ebro, la ciudad parece configurarse desde los primeros momentos como punto de encuentro de la población romana e indígena de Salduie.

Del periodo de ocupación más antiguo del solar de la calle Don Juan de Aragón, se conservan un suelo y algunas depresiones - hogares y silos-que fueron localizados bajo el pavimento de opus signinum. La presencia en un mismo espa- 


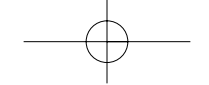

cio del hogar y el almacén sugieren que la habitación tuvo la función de cocinadespensa. El suelo es de tierra batida mezclada con gravilla y alcanza un grado de dureza considerable. En él se documentaron tres silos y un hogar formado por los hombros de un ánfora grecolatina (Galve 1996: 33-36).

De la estancia triclinar, perteneciente a una segunda fase del solar, se exhumaron más de $30 \mathrm{~m}^{2}$ de opus signinum y dos muros de cierre: el occidental y el septentrional (Galve 1996: 36). El esquema compositivo del pavimento es bastante claro para definirlo como un triclinio. Para Galve (1996: 36) se trataría de un comedor de invierno, siguiendo las normas vitruvianas, por su acceso orientado hacia el suroeste. Respecto a su ubicación dentro de la casa, ha sido imposible detectarlo, ya que es la única habitación que se ha conservado de la vivienda.

Sin embargo, en la zona sur del solar se documentaron una serie de estancias que pertenecieron a la misma época que la del triclinio. Se trataría de espacios adyacentes documentados en la fase final de Azaila o Alto Chacón que sugieren la utilización de estas estancias para trabajos agrícolas de transformación y, de igual modo, como cocina-despensa. Los distintos apartamentos de forma rectangular están adosados unos a otros, constituyendo quizás viviendas independientes. Los hogares aparecen en todas las estancias excavadas, e incluso en algunas de ellas había más de uno, casi todos próximos a los muros y preferentemente en zonas angulares. También, se documentó en otra habitación una pileta de yeso con abundantes fragmentos de una olla hecha a mano de tradición hallstáttica. Por todos estos datos, Galve llega a la conclusión de que pudo tratarse de habitaciones familiares de tipo indígena (1996: 56-61).
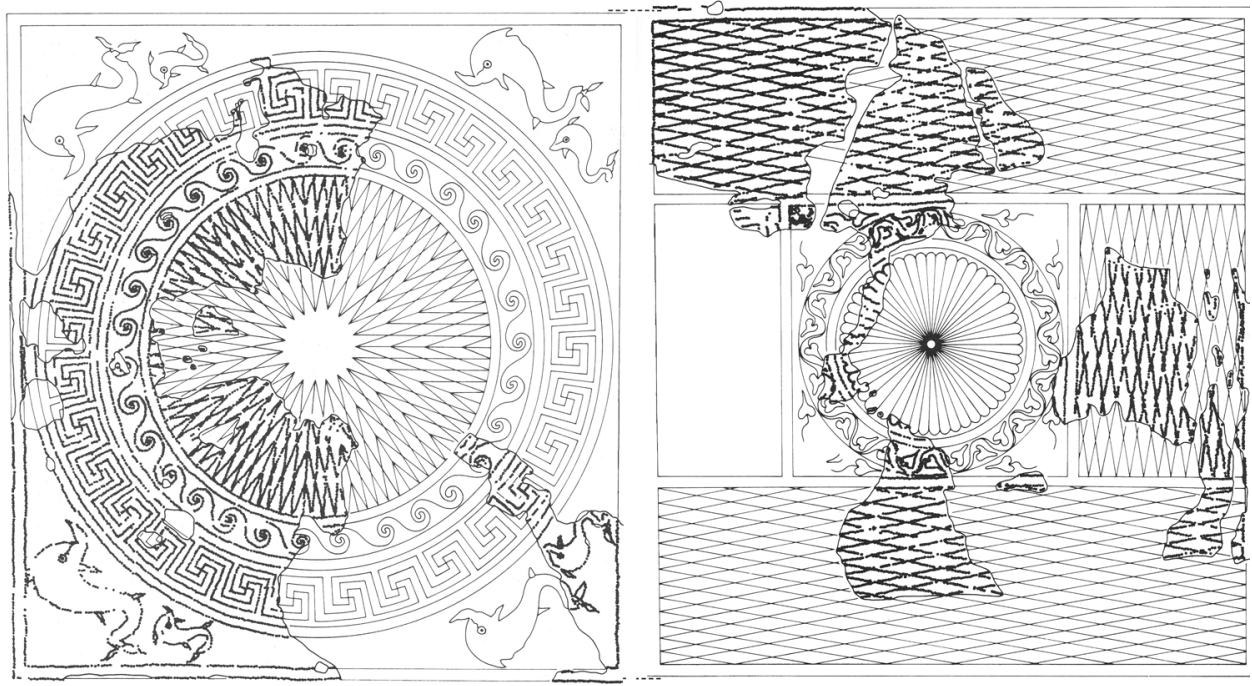

Fig. 5. Opus signinum de la vivienda romana de la C/ Don Juan de Aragón, 9, Caesar Augusta (a partir de Galve 1996). 
Los fenómenos de imitación en las viviendas urbanas romanas en el Nordeste...

De esta manera, Galve (1996: 56) afirma que sus habitantes fueron indígenas «con un fuerte impacto colonial itálico», relacionándolos incluso con aquéllos a los que se les concedió la ciudadanía en el Bronce de Ascoli (89 a.C.).

\section{BILBILIS (CALATAYUD, ZARAGOZA)}

Finalmente, presentamos un ejemplo, la domus 1 de Bilbilis, que demuestra que, en momentos avanzados cronológicamente, diferenciar entre la vivienda de un indígena romanizado, perteneciente a una clase social media-alta, y un colono itálico también de clase media asentado en nuestro territorio, resulta tarea ardua y casi imposible. Ubicada a las orillas del río Jalón, la ciudad de Bilbilis tuvo su origen en una transductio, pasando de ciudad indígena a denominarse, desde inicios del I a.C., Bilbilis Italica con el asentamiento de un contingente itálico desde estas fechas. Escenario del enfrentamiento entre Sertorio y Metelo primero y César y Pompeyo después, su adscripción al bando cesariano le permitió una rápida ascensión al rango municipal en época de Augusto. Desde este momento se planifica una ciudad netamente romana con un gran centro monumental, culminado en época tiberiana y ampliado con Trajano (Martín-Bueno 1975; 1987; 2000).

La domus I se estructura en torno a un atrio tetrástilo que se construye sin impluvio central, hecho que algunos autores han interpretado como una consecuencia de la emulatio. Para Beltrán (2003: 23), la ausencia de impluvio hace pensar en la adaptación del espacio del atrio a un sistema de patio. Es decir, un atrio desprovisto de su función principal adoptado más bien como un símbolo de prestigio, al igual que en la ínsula del Vaso Fálico en Conimbriga. Según Gros (2001: 207), esta estructura totalmente desprovista de su funcionalidad inicial —recoger aguase ubicaría en la vivienda de un petit bourgeois como símbolo de prestigio y romanidad ${ }^{4}$.

Sin embargo, en el caso de la vivienda bilbilitana, el símbolo de su marcado carácter ya no romano sino campano, viene subrayado por la presencia de un antiguo balneum, perteneciente a un grupo primitivo del desarrollo termal, de evidente similitud con los ejemplos campanos y fechado en el cambio de era 5 . En nuestra opinión, compartida por Garcia-Entero (2005: 274-78), el propietario de la vivienda estaría más relacionado con un origen campano ${ }^{6}$ que local.

\footnotetext{
${ }^{4}$ Como símbolo también de romanidad se edificaron en la Grecia romana las casas constituidas en torno a un atrio tetrástilo con impluvio. Según Bonini (2006: 58), la presencia de estos patios tetrástilos manifiesta en Grecia una verdadera praxis constructiva romana, correspondiente a una clase media de colonos occidentales que se asentaron en las colonias griegas.

5 El balneum de la domus I de Bilbilis estuvo constituido por una bañera de obra y el hueco para la letrina. Destaca, por su rareza, que ambos estuvieron sitos en el mismo espacio, además de contar con un hypocaustum que permitía calentar la habitación. Sobre esta instalación termal doméstica, ver MartínBueno, Reklaityte, Saénz y Uribe (2007).

6 Relacionando estas dos ideas se nos plantea una pregunta, en cierto modo retórica, sobre la tipología de la vivienda. Si asumimos que el propietario fuese un campano asentado en el municipio, ¿por qué, conociendo las soluciones de atrios tetrástilos con impluvio, realizó la obra de este modo? Este hecho puede que tuviese que ver con dos causas que, por el momento, somos incapaces de determinar. La
} 


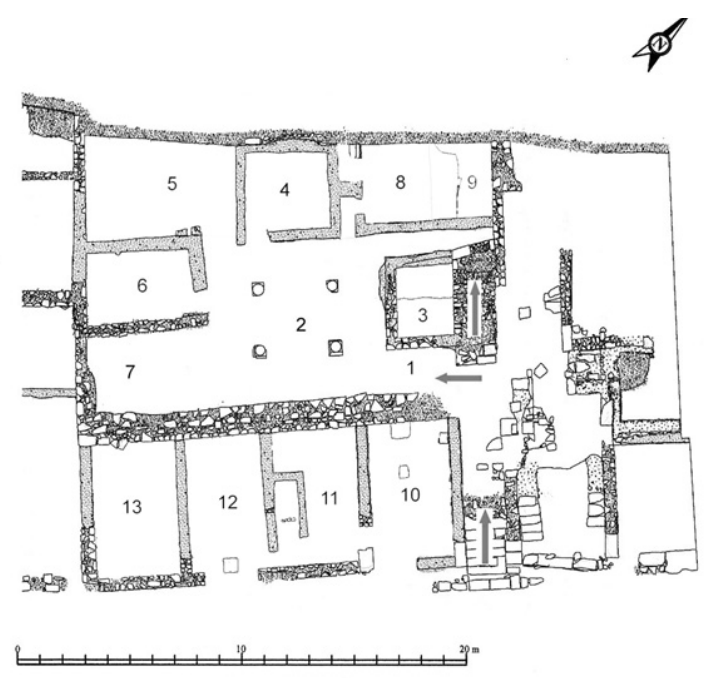

Figura 6. Plano de la Domus I de Bilbilis (a partir del dibujo realizado L. Lanteri y C. Vacarella, inédito).

\section{CONCLUSIONES}

A través de estos cuatro ejemplos de mimesis podemos concluir que en la edilicia doméstica no se imitan únicamente determinados objetos, prácticas o costumbres, sino que en realidad, al poseer o vivir en una vivienda 'a la romana' no sólo se imita un modelo arquitectónico, sino que se asume por completo el modelo de vida del colonizador. En definitiva, en este caso se imitan estos modelos porque existe una voluntad expresa, sobre todo por parte de las elites locales, de vivir a la manera romana.

Las imitaciones que hemos expuesto son bastante fieles a los parámetros itálicos. Sin embargo, estos modelos importados a la Península sufren determinadas adaptaciones según las necesidades del momento. Es decir, dependían del solar que se disponía para construir, de la capacidad económica del propietario y de los gustos personales. En este sentido, se puede documentar cómo la fórmula de patio porticado que observamos en Azaila o en La Caridad se repite, en el Nordeste de la Península Ibérica, en la Casa del Pretorio de Arcobriga en un momento avanzado del I d.C., con un patio central con 12 columnas, aunque de menores dimensiones que Likine.

primera de ellas es la ya apuntada por Beltrán, aquélla en la que un atrio tetrástilo, desprovisto de su función principal, daría simplemente monumentalidad a la vivienda. Sin embargo, nos planteamos si, suplidas las necesidades de abastecimiento de agua - a través de la conocida red de cisternas - junto con las dificultades de excavar en profundidad por el afloramiento de la roca, la presencia de un impluvium fuese prescindible. 
Los fenómenos de imitación en las viviendas urbanas romanas en el Nordeste...

La misma configuración estructural se observa también en las casas de los Morillos y la Llanuca 2 de luliobriga. La primera de ellas se constituyó como un patio porticado con dieciséis columnas, pero no queda clara la existencia de un impluvio. Fernández (1993: 78) afirma que no tenía, y describe un aljibe o cisterna central descubierto, hecho de arcilla (1999: 209). Para, Beltrán (2003: 27, n.55) no se trataría de un atrio corintio debido a que careció de impluvium, su patio no estuvo enlosado y tampoco poseyó cisterna para el agua. En el segundo caso, la Casa de la Llanuca 2, sí que se documentó una cisterna situada en el lado oeste del patio, sin embargo el pórtico no estuvo sustentado por columnas, sino por un muro corrido (Fernández 1993: 122-23).

Cabría añadir a este grupo, aunque no estén excavadas en su totalidad: la vivienda descubierta en el solar de la C/ Alguer de Tarragona, con una fuente ubicada en el patio en época antoniniana, la denominada Casa del Peristilo de Andelos (Mezquíriz 1987: 181), la Casa $n .^{\circ} 3$ de Clunia (Palol 1994: 61), y el ejemplo de vivienda con espacio central columnado de Numancia (Jimeno et alii 2002: 11011). De este modo, podemos suponer una transformación del propio modelo, relacionado con el sentido más amplio de la casa mediterránea -aquélla que se configura en torno a un patio abierto- documentada, también en otras zonas del Imperio, como por ejemplo en la provincia gala o en el norte de Italia ${ }^{7}$.

Respecto a los elementos que se ha elegido imitar y en los cuales se ha preferido mantener fórmulas locales, un claro ejemplo del profundo arraigo del modus vivendi romano son los triclinios de verano, salones de aparato, balnea o lararios documentados en el Nordeste de la Península. No obstante, y al igual que se observa en el resto de las provincias, estas viviendas difieren en ciertos aspectos de las residencias itálicas. Así, los tablina se encuentran en escaso número, y elementos característicos como, por ejemplo, los bancos de las cocinas campanas o los de obra de los triclinia todavía no se han podido documentar arqueológicamente.

En cuanto al contexto cronológico de la imitación, podríamos decir, si tomamos como referencia los ejemplos de la Caridad y de Caesar Agusta, que las primeras imitaciones surgen ya en el I a.C. e incluso a finales del II a.C., consecuencia, obviamente, de la temprana colonización de nuestra Península y de la política de asentamiento de itálicos en los territorios ocupados desde los primeros momentos de la conquista. Así, los fenómenos de imitación en la edilicia doméstica del nordeste peninsular estarían estrechamente relacionados con el establecimiento de las primeras generaciones de colonos, quienes trasladan sus modelos edilicios privados a las nuevas ciudades romanas, como es el caso de las Casas 1, 2A y 2B de Ampurias (Santos 1991). Finalmente, en el caso de la edilicia doméstica del Nordeste peninsular, se imitarían principalmente los modelos de la metrópoli. Éstos, a pesar de los conceptos historiográficos simplistas y anquilosados de casas itálicas y casas pompeyanas, fueron mucho más complejos y variados.

7 Véase: Delos (Brunneau 1972: 146), en la Magna Grecia en Locri Epizefiri (Barra 1996:50), en Italia en Cosa (Bruno y Scott 1993), en la Emilia-Romagna — con escasa recepción del modelo de peristilo a favor de un área de patio- (Scagliarini 1983: 316), además de en Glanum o Bibracte (Beltrán 1991: 135). 


\section{BIBLIOGRAFÍA}

CABRÉ, J. (1925), Los Bronces de Azaila, AEA 3, Madrid.

- (1940), La Acrópolis de Alcalá, Teruel, AEA 14, Madrid,

BALIL, A. (1971), “Casa y urbanismo en la España antigua», BSAA 37, 311-328.

- (1972), "Casa y urbanismo en la España antigua», BSAA 38, 55-131.

- (1973), "Casa y urbanismo en la España antigua», BSAA 39, 115-188.

BARRA, M. (1996), "La casa in Magna Grecia», en D'Andria, F y Mannini, K. eds. Richerche sulla Casa in Magna Grecia e in Sicilia. Atti del Coloquio à Lecce, Archeologia e Storia 5, 41-66.

BELTRÁN, M. (1976), Arqueología e Historia de las Ciudades Antiguas del Cabezo de Alcalá de Azaila. Monografías Arqueológicas 19, Zaragoza.

- (1984), «Nuevas aportaciones a la cronología de Azaila», MZB 3. 125-152.

- (1991), «La Colonia Celsa», en Actas de la Casa Urbana Hispanorromana, Zaragoza, 131-164.

- (2003), «La casa hispanorromana. Modelos», Bolskan, 20, 13-63.

- ed. (2007), Ciudades Romanas de Hispania. Las Capitales Provinciales: Zaragoza, CoIonia Caesar Agusta, Zaragoza.

BELTRÁN, M y FATÁS, G. (1998), Caesaraugusta. Ciudad Romana. Historia de Zaragoza, 2, Zaragoza.

BONINI, P. (2006), La Casa nella Grecia Romana. Forme e Funzioni dello Spazio Privato fra I e VI Secolo, Antenor-Quaderni 6, Roma.

BRUNNEAU, P. (1972), Exploration Archéologique de Délos. Les Mosaiques, Paris.

BRUNO, J. y SCOTT, T .R. (1993), Cosa IV. The Houses, Rome.

FERNÁNDEZ VEGA, P. A. (1993), Arquitectura y Urbanística en la Ciudad Romana de Julióbriga, Santander.

- (1999), «Lares y hogares: la casa cántabro-romana de los Morillos en luliobriga», en Iglesias, J. M. y Muñiz, J. A. eds., Regio Cantabrorum, Santander, 205-214.

- (2000), «La casa de atrio en la España romana», en Hernández, L; Salcedo, A. y Solana, M. J. eds., Actas del I Congreso Internacional de Historia Antigua. La Península Ibérica hace 2000 años, Valladolid, 681-697.

GALVE, M. P. (1991), «¿Salduie en el centro histórico de Zaragoza? Hallazgo de estructuras iberorromanas», en Actas de la Casa Urbana Hispanorromana, Zaragoza, 203-209.

- (1996), Los Antecedentes de Caesaraugusta. Estructuras Domésticas de Salduie, Zaragoza.

GARCIA-ENTERO, V. (2005), Los Balnea Domésticos (Ámbito Rural y Urbano) en la Hispania Romana, Anejos de AEspA 37, Madrid.

GROS, P. (2001), L'Architecture Romaine II: Maisons, Palais et Tombeaux, Paris.

JIMENO, A. et alii (2002), Numancia (Garray, Soria), Soria.

MARTÍN-BUENO, M. y SAENZ, C. (2001-2002), «La ínsula I de Bilbilis», Salduie 1, 127-158.

MARTÍN-BUENO, M; SÁENZ, C; REKLAITYTE, I. y URIBE, P. (2007), «Baños y letrinas en el mundo romano: el balneum de la domus I, ínsula de las Termas, Municipium Augustam Bilbilis (Calatayud)», Zephyrus 60, 205-223.

MEZQUÍRIZ, M. (1987), «La ciudad de Andelos. Secuencia estratigráfica y evolución cronológica», en I Congreso General de Historia de Navarra, Príncipe de Viana 48, Anejo 7, 517-530.

PALOL, P. (1959), Clunia Sulpicia. Ciudad Romana. Su Historia y Presente, Burgos.

- (1965), «Notas de arqueología cluniense», Pyrenae 1, 181-184.

- (1994), Clunia. Historia de la Ciudad y Guía de las Excavaciones, Burgos.

RUSSO, A. (1996), «Le abitazioni degli indigeni: problematiche generali», en D'Andria, F y Mannini, K. eds., Richerche sulla Casa in Magna Grecia e in Sicilia. Atti del Coloquio à Lecce, Archeologia e Storia 5, 67-87.

SANTOS, M. (1991), «Distribución y evolución de la vivienda urbana tardorrepublicana y altoimperial en Ampurias», en Actas de la Casa Urbana Hispanorromana, Zaragoza, 19-34. 
Los fenómenos de imitación en las viviendas urbanas romanas en el Nordeste...

- (1998), «Una aproximación al estudio de la arquitectura doméstica en la Ampurias tardorrepublicana», en Mayer, M; Nolla, J. M.; Pardo, J. eds., De les Estructures Indígenas a l'Organització Provincial Romana de la Hispana Citerior, Barcelona, 555-565.

SCAGLIARINI, D. (1983), “L'edilizia residénciale nelle cittá romane dell'Emilia Romagna», en Studi sulla città antica. L'Emilia-Romagna, Roma, 283-334.

URIBE, P. (2004), «Arquitectura doméstica en Bilbilis, la domus I», Salduie 4, 191- 220.

VICENTE, J.; MARTÍN, R; HERCE, A. I; ESCRICHE, C. y PUNTER, P. (1989), «Un pavimento de opus signinum con epígrafe ibérico", en Mosaicos Romanos in Memoriam Manuel Fernández-Galiano, 11-42.

VICENTE, J.; PUNTER, M. P. (1988-1989), «Informe sobre la VII campaña de excavaciones arqueológicas en La Caridad (Caminreal, Teruel)», Arqueología Aragonesa 11, 183-187.

VICENTE, J.; PUNTER, M. P. y EZQUERRA, B. (1997), "La catapulta tardo-republicana y otro equipamiento militar de La Caridad (Caminreal, Teruel)», JRMES 8, 167-199.

VICENTE, J.; PUNTER, M. P.; ESCRICHE, C y HERCE, A. (1989), «El mosaico romano con inscripción ibérica de la La Caridad (Caminreal, Teruel)», Xiloca 3, 9-29.

- (1986), La Ciudad Celtibérica de La Caridad (Caminreal, Teruel), Teruel.

- (1991), «La Caridad (Caminreal, Teruel)», en Actas de la Casa Urbana Hispanorromana, Zaragoza, 81-130.

- (1993), «Las inscripciones de la Casa de Likine», en Untermann, J. y Villar, F. eds., Lengua y Cultura en la Hispania Prerromana, Salamanca, 747-772. 